\title{
Giant Retroperitoneal Schwannoma: A Case Treated with 3D Laparoscopic Resection
}

\author{
Heng Chieh Chiang, Meng Yi Yan, Chao An Chen and Pao Hwa Chen* \\ Department of Surgery, Changhua Christian Hospital, Changhua, Taiwan \\ Received: 㭗 August 18, 2018; Published: 海 August 22, 2018 \\ *Corresponding author: Pao Hwa Chen, Department of Surgery, Division of Urology, Changhua Christian Hospital135, Taiwan
}

\section{Abstract}

Background: Schwannomas which are derived from Schwann cell and strictly found in head or neck region, upper and lower limbs. Retroperitoneal location accounts for less than $3 \%$ of primary schwannoma. We present a case of huge retroperitoneal schwannoma treated using 3D laparoscopy.

Case Report: A 36 years-old gentleman was referred to our clinic due to suspected renal lesion. After imaging, a huge retroperitoneal tumor was noted on CT scan. Due to suspected sarcoma, surgical intervention was performed. The final pathology showed Antoni A and B section along with S100 and SOX-10 positive, therefore a diagnosis of benign schwannoma was given.

Discussion: A variety of diagnosis can arrive from retroperitoneum tumor, ranging from sarcoma to lymphoma. The size of tumor, the clinical symptoms, the involvement of peripheral organ along with selective pre-operative biopsy will help determine the treatment course of these patients. Pre-operative biopsy should be considered in selected individuals to help with decision making and avoid any unnecessary surgery.

Conclusion: A careful pre-operative survey and selective biopsy is warranted for patients with retroperitoneal tumor.

Keywords: 3D Laparoscopic Resection; Retroperitoneal Mass; Retroperitoneal; Schwannoma; Trans Peritoneal

Abbreviations: XGP: Xantho Granulomatous Pyelonephritis; KUB: Kidney Ureter Bladder X-ray; CT Scan: Computed Tomography Scan

\section{Introduction}

Schwannomas, previously known as neurilemmoma, are neuroectodermal tumors derived from Schwann cell of nerve sheaths and nearly $75 \%$ of schwannomas are found in head or neck region, upper limbs and lower limbs. Cranial nerve I and II are spared due to lack of Schwann cells. Retroperitoneal schwannoma accounts for less than $3 \%$ of primary schwannoma and less than $6 \%$ of all retroperitoneal tumor [1-5]. Retroperitoneal mass has a wide variety of pathology ranging from sarcoma (liposarcoma, leiomyosarcoma), lymphoproliferative (Hodgkin's), epithelial (kidney, adrenal, pancreas), metastatic (germ cell tumor, melanoma), benign (schwannomas, paragangliomas, angiomyolipoma). Pre-operative imaging is helpful in determining the possible etiology and useful during patient consultation during shared decision making. The choices of treatment depend on pre-operative tentative diagnosis, size and location of tumor, and surgeon preference. In cases of unsure pathology, different studies express different opinion on the use of percutaneous biopsy [3-9]. We present a case of giant retroperitoneal schwannoma treated with 3D laparoscopic resection.

\section{Case Presentation}

A 36 years-old gentleman (Height: $180 \mathrm{~cm}$, Body weight: $130 \mathrm{~kg}$ ) with history of hypertension was referred to our Urology department at Changhua Christian Hospital due to abnormal left side renal ultrasound. The patient had a routine health exam and abnormal left side kidney was noted and the initial urologist had an initial impression of xantho granulomatous pyelonephritis (XGP). Since the 5patient did not provide images from his health exam, a baseline ultrasound and kidney-ureter-bladder x-ray (KUB) as performed during clinic hours (Figure 1). Right side kidney was readily visible and left side mixed echogenicity mass was noted butthe left side kidney was not visible. The KUB was unremarkable for any urolithiasis, which is a hallmark for XGP. Computed tomography scan (CT scan) was ordered to check for origin of the mass. CT scan showed a retroperitoneal mass extending from Psoas muscle to the lower portion of adrenal glands. The mass seems to be growing from Psoas muscle and extending upward adhering on the lower pole of kidney (Figure 2). 


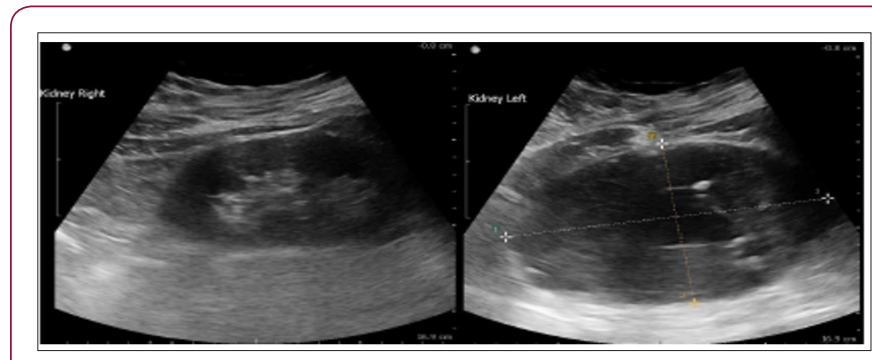

Figure 1: Pre-OP sonography, Right kidney visible but left retroperitoneal $9 \times 15 \mathrm{~cm}$ Mass.

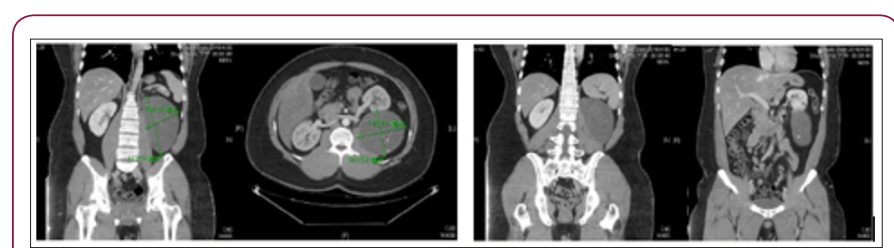

Figure 2: Abdominal CT scan.

After discussing with radiologist, initial impression including retroperitoneal sarcoma or neurogenic tumor. After discussing initial finding with the patient and considering the size of tumor, hand-assisted trans-peritoneal laparoscopic approach would provide the most minimally invasive method and the patient agree to undertake operation. Pre-operative survey, Operative method, and Post-Operative care After general anesthesia, the patient is placed on lateral decubitus position with a flat table and secured with masking tape with all the joints well padded. A $7 \mathrm{~cm}$ midline incision around the umbilicus was done for the Gel port (Applied Medical, Rancho Santa Margarita, California). Two $12 \mathrm{~mm}$ trocar was used for instrumentation and camera port for the 3D endoscopy (Olympus Taiwan Co., Ltd). Since the tumor is near the lower pole of kidney, the left side colon was taken down similar approach as radical nephrectomy. After freeing up colon, the tumor can easily be seen and felt [6]. As the pre-operative imaging showed, the tumor was not adhered to the colon and can easily be separated. Fortunately, the tumor was not adhered to the lower pole of kidney and can easily be separated with the tumor capsule intact.

The only adhesion portion is the posterior part near the iliac crest. Careful hand dissection with the combination of Liga Sure laparoscopic sealer/divider (Medtronic: Minneapolis, Minnesota) ensure a safe resection without capsule compromise (Figure 3). Due to pre-operative imaging suggestive of sarcoma, an intraoperative frozen section was sent from the Psoas muscle after the tumor was removed which does not show any malignant pathology. The tumor was removed from the Gel port wound and wound was closed after a drain was inserted at retroperitoneal space. The gross examination showed yellowish with jelly-like component (Figure 3). Microscopically showed a well encapsulated tumor with loosely arranged spindle cells with wavy nuclei. Stroma is myxoid with edematous area alternating with fibrotic or hyalinizing area with focal calcification. Immunohistochemical study showed S-100 and Sox-10 positive (Figure 4). The overall picture highly suggestive of schwannoma. The patient was asked to start taking clear liquid after transferring to ward from the post-operative recovery room and slowly increase oral diet at his own pace. He started full diet on post-operative day 2 and discharged on post-operative day 5 after removing the retroperitoneal drain.

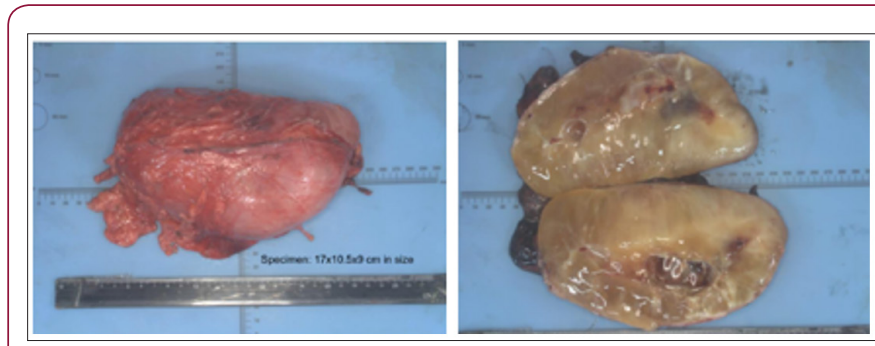

Figure 3: Gross pathology without any capsule compromise.

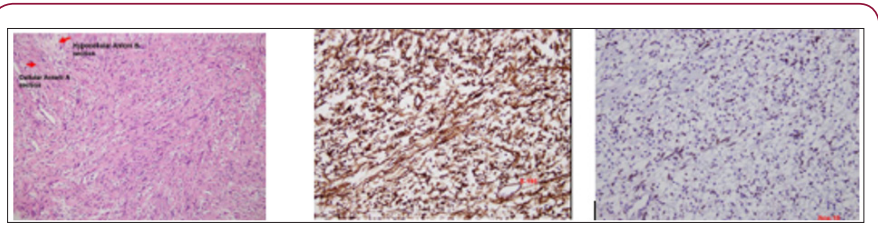

Figure 4: Cellular and hypocellular section of Antoni A and B, S100 and SOX-10 stain positive.

\section{Discussion}

Schwannomas are relative benign mass mostly recurring in head or neck and upper 7 limbs. In cases of reported retroperitoneal schwannomas, the tumors are usually large (average $9 \mathrm{~cm}$ in diameter) and asymptomatic. Due to the large malleable space in the retroperitoneal, retroperitoneal tumors are usually asymptomatic and found incidentally during examinations. The most common symptoms are attributed to the tumor size affecting the nearby systems (palpable mass, non-specific abdominal discomfort, urinary difficulties and frequency) $[2,3,9,10]$. The key step in treating patients with retroperitoneal mass of unknown pathology is deciding whether to undergo surgical resection. For large, symptomatic tumor or patients with known malignancy in which metastasectomy is indicated, the decision to remove the retroperitoneal tumor would be easier to decide. On the other hand, an incidentally found tumor of relative size $(<10 \mathrm{~cm})$ without any symptoms and pre-operative imaging favors benign pathology, surgical removal of the tumor could cause unwanted complications for the patients. Li et al. [2] reported 82 cases of retroperitoneal schwannoma had complications such as massive bleeding (2500cc blood loss), femoral nerve injury causing quadriceps femoris paralysis, and 1 mortality due to anesthesia complications [9].

Percutaneous biopsy seems to be another point of discussion among doctors encountering patients with retroperitoneal tumor. Some doctors are against biopsy due to possibility of biopsy related complications (bleeding, infection, tumor seeding). On the other hands, Strauss et al. [3] managed 8 patients conservatively due to benign biopsy results. Biopsy could also change course of treatment due to the chemo sensitive characteristic of some tumor 8 (Ewing's sarcoma, GIST) which can result in neo-adjuvant treatment [3]. For large symptomatic tumor without any involvement to surrounding organs, surgical decision should be the first consideration. For tumors with peripheral organ involvement, percutaneous biopsy should be considered since it provides information for 
healthcare givers and patients to reach a joined consent. For small asymptomatic incidental finding tumor, biopsy should also be considered since benign cases could be managed conservatively. Therefore, a careful pre-operative evaluation and share decision making during patient consultation is vital to ensure the well-being of the patients.

\section{Ethics Approval and Consent to Participate}

All procedures performed in studies involving human participants were in accordance with the ethical standards of the institutional and/or national research committee and with the 1964 Helsinki declaration and its later amendments or comparable ethical standards.

\section{References}

1. Whitaker WG DC (1976) Benign encapsulated neurilemmoma: a report of 76 cases. Am Surg 42(9): 675-678.

2. Li Q, GC (2006) Clinical Analysis of 81 Cases of Benign Retroperitoneal Schwannoma. The Chinese-German Journal of Clinical Oncology 5(1) 28-30.

3. Strauss DC, QY, Hayes AJ, Thomas JM (2011) Management of benign retroperitoneal schwannomas: A single-center experience. American journal of surgery 202(2): 194-198.

\section{ISSN: 2574-1241}

DOI: 10.26717/BJSTR.2018.08.001626

Pao Hwa Chen. Biomed J Sci \& Tech Res

(c) (P) This work is licensed under Creative

Submission Link: https://biomedres.us/submit-manuscript.php
4. Maruyama T, SY Miyagishima D, Toru Fukunaga, Kentaro Tasaki, Nobuyoshi Takeshita, et al. (2015) Laparoscopic resection of a retroperitoneal schwannoma located in the hepatic hilus. Surgical case reports 1: 18 .

5. Tortorelli AP, RF, Papa V, Rotondi V, Sanchez AM, et al. (2007) Retroperitoneal schwannomas: Diagnostic and therapeutic implications. Tumori 93(3): 312-315.

6. Hoarau N, SK, Da Ines D (2013) CT and MR imaging of retroperitoneal schwannoma. Diagnostic and interventional imaging 94(11): 11331139.

7. Fujimoto N, KT, Hisaoka M, Udo K, Yokomizo A, et al. (2018) Demographics, management and treatment outcomes of benign and malignant retroperitoneal tumors in Japan. International Journal of Urology 25(1): 61-67.

8. Hughes MJ, TJ, Fisher C, Moskovic EC (2005) Imaging features of retroperitoneal and pelvic schwannomas. Clin Radiol 60 (8): 886-893.

9. Li Q GC, Juzi JT, Hao X (2007) Analysis of 82 cases of retroperitoneal schwannoma. ANZ journal of surgery 77(4): 237-340.

10. Gubbay AD, MG, Gray BN, Thompson I (1995) Retroperitoneal schwannoma: a case series and review. Aust N Z J Surg 65(3): 197-200.

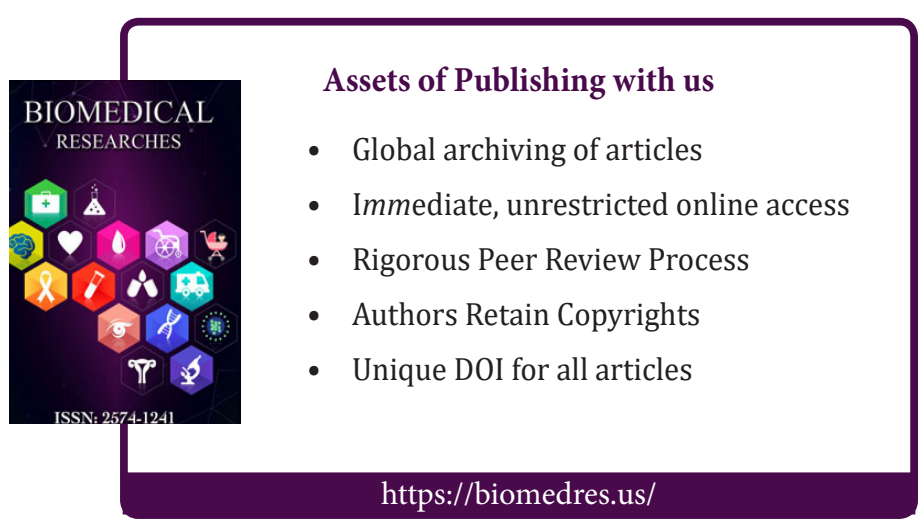

